\title{
Níveis críticos de fósforo em solos cultivados com cana-de-açúcar em Pernambuco ${ }^{1}$
}

\author{
Djalma Euzébio Simões Neto ${ }^{2}$, Alexandre Campelo de Oliveira ${ }^{3}$, Alexandre Tavares da Rocha ${ }^{4}$, Femando José \\ Freire $^{5}$, Maria Betânia Galvão dos Santos Freire ${ }^{5}$, Clístenes Williams Araújo do Nascimento 5
}

\section{RESUMO}

Em Pernambuco, a disponibilidade de P é avaliada sem se considerarem características físicas, químicas, ou, mineralógicas dos solos. Este trabalho teve como objetivos determinar os níveis críticos de $\mathrm{P}$ em diferentes solos, utilizando-se diferentes extratores químicos, considerando-se a quantidade e a qualidade da fração argila e relacionar as características físicas, químicas e mineralógicas dos solos com os níveis críticos de P. Os solos foram classificados como: Argissolo Amarelo distrocoeso (PAdx); Latossolo Amarelo distrófico (LAd); Argissolo Amarelo distrófico (PAd); Gleissolo háplico eutrófico (GXve) e Espodossolo cárbico duripânico (EKo). Os tratamentos consistiram em sete doses de $\mathrm{P}$, determinadas de acordo com a capacidade máxima de adsorção de P (CMAP). O delineamento experimental foi em blocos ao acaso, com quatro repetições. Foram coletadas amostras de solo na área útil das parcelas. Essa amostragem foi realizada 30 dias após o plantio e aplicação das doses de P, numa profundidade de 0,0-0,2 m. Nessas amostras, determinaram-se o teor de P pelos extratores Mehlich-1, Mehlich-3, Bray-1 e Resina de Troca Iônica (RTA). Com os teores de P no solo e a produção agrícola, determinaram-se os níveis críticos de P no solo. A RTA extraiu mais P independentemente do tipo de solo. Os níveis críticos de P no solo foram dependentes da CMAP, da quantidade de argila e do P remanescente (P-rem), quando o extrator para avaliação do P disponível foi o Mehlich-1. Os níveis críticos no solo determinados pela RTA não foram dependentes de nenhuma característica química e, ou, física dos solos.

Palavras-chave: Adsorção de P, disponibilidade de P, extratores de P.

\section{ABSTRACT}

\section{Critical levels of phosphorus in sugarcane soils of Pernambuco State, Brazil}

Physical, chemical and mineralogical properties of soils are not observed to estimate phosphorus $(\mathrm{P})$ availability in Pernambuco, Brazil. The aim of this work was to determine phosphorus critical levels in distinct soils, using different chemical extractors, considering clay fraction content and quality, and to relate soil physical, chemical and mineralogical characteristics with P critical levels. The soils were classified like: Typic Haplustults; Typic Hapludox; Typic Hapludults; Typic Hydraquents and Typic Haplohumods. Treatments consisted of seven P doses, determined in function of $\mathrm{P}$ maximum adsorption capacity (PMAC). The experimental design was in randomized blocks, with four replicates. Samples of soil were collected at lots useful area. This sampling was made 30 days after the plantation and treatments application at 0,0-0,2 m depth. In these samples it was determined $\mathrm{P}$ content by the extractors Mehlich-1, Mehlich-3, Bray-1 and

Recebido para publicação em 23/11/2010 e aprovado em 10/11/2011

${ }^{1}$ Parte da Tese de Doutorado apresentada pelo primeiro autor à UFRPE. Pesquisa financiada pelo CNPq.

Engenheiro-Agronômo, Doutor. Universidade Federal Rural de Pernambuco. Estação Experimental de Cana-de-açúcar de Carpina, Rua Ângela Cristina de Luna, s/n. 55.819255, Carpina, Pernambuco, Brasil. desn@oi.com.br

${ }^{3}$ Engenheiro-Agronômo, Doutorando. Universidade Federal Rural de Pernambuco, Departamento de Agronomia, Rua Dom Manoel de Medeiros, s/n. 52.171-900, Recife, Pernambuco, Brasil. alexcampelo@oi.com.br

${ }^{4}$ Engenheiro-Agronômo, Doutor. Universidade Federal Rural de Pernambuco, Unidade Acadêmica de Serra Talhada, Fazenda Saco, s/n. 56.900-000, Serra Talhada, Pernambuco, Brasil. alexandre@uast.ufrpe.br

${ }^{5}$ Engenheiros-Agronômos, Doutores. Universidade Federal Rural de Pernambuco, Departamento de Agronomia, Rua Dom Manoel de Medeiros, s/n. 52.171-900, Recife, Pernambuco, Brasil. f.freire@depa.ufrpe.br; betania@depa.ufrpe.br; clistenes@depa.ufrpe.br

Rev. Ceres, Viçosa, v. 58, n.6, p. 802-810, nov/dez, 2011 
Ionic Change Resin (ICR). The soil P critical levels were determined with soil P content and agricultural production data. Indistinctly, ICR extracted more P, independently of soil. Soil P critical levels were dependent from PMAC, clay content and P-remaining (P-rem), when the extractor to $\mathrm{P}$ available estimation was Mehlich-1. Soil critical levels determined by ICR were not dependents of soil chemical and or physical characteristics.

Key words: P adsorption, P availability, P extractors.

\section{INTRODUÇÃO}

O Brasil é, atualmente, o maior produtor mundial de cana-de-açúcar e ocupa uma área de 7,5 milhões de hectares, com uma produtividade média de 81,9 tha $^{-1}$ (CONAB, 2009). No Nordeste, a produtividade média na safra 2008/ 2009 foi de $61,2 \mathrm{tha}^{-1}$, ou seja, cerca de $20 \mathrm{tha}^{-1}$ a menos, em relação à média nacional. Parcela significativa dessa redução de produtividade é atribuída à pouca assistência que se tem dado a pesquisas relacionadas com a fertilidade do solo e com a nutrição de plantas. Particularmanete em Pernambuco, a disponibilidade de Pé avaliada sem se considerarem características físicas, químicas e, ou, mineralógicas dos solos. Essa avaliação pode estar sub ou super- estimando as recomendações de adubação fosfatada no Estado e contribuindo, significativamente, para a diminuição da produtividade da cultura.

Os solos tropicais são naturalmente pouco férteis, principalmente quando essa fertilidade está relacionada com a disponibilidade de P. A recomendação de doses adequadas desse nutriente deve ser baseada na utilização de métodos que extraiam o $\mathrm{P}$ disponível do solo, para que se possam estabelecer os níveis críticos do nutriente, para uma determinada produtividade esperada e economicamente viável. Segundo Rossi (1995), o nível crítico de um nutriente no solo é o teor mínimo, abaixo do qual há grande possibilidade de resposta a sua aplicação e, acima do qual, essa probabilidade diminui.

Os primeiros ensaios com cana-de-açúcar, no sentido de encontrar um extrator de $\mathrm{P}$ do solo para diagnosticar a necessidade de adubação, foram realizados no Brasil por Strauss (1954), que comparou os teores totais do elemento com as frações extraídas por $\mathrm{KOH} 0,5 \mathrm{~mol} \mathrm{~L}^{-1}$ ou por $\mathrm{HCl}$ 0,5 $\mathrm{mol} \mathrm{L}^{-1}$, na época, usados no Havaí. Nesses ensaios, esse autor obteve boas correlações com a produção relativa da cana-de-açúcar, sugerindo, ainda, o emprego da relação entre o P do solo e o teor de argila, para melhorar a confiabilidade da recomendação.

Segundo Silva (1991), na década de 70, no Estado de São Paulo, o extrator mais utilizado para aferir as necessidades da adubação fosfatada era a solução de $\mathrm{H}_{2} \mathrm{SO}_{4} 0,025$ $\mathrm{mol} \mathrm{L}^{-1}$ e, no restante do País, o extrator Mehlich-1. Posteriormente, Bittencourt et al. (1978) propuseram o uso de uma solução mais concentrada $\left(\mathrm{H}_{2} \mathrm{SO}_{4} 0,25 \mathrm{~mol} \mathrm{~L}^{-1}\right)$ para medir o P disponível, em São Paulo, provavelmente pela maior capacidade de extração desta solução e sua melhor correlação com a resposta da cana-de-açúcar. Pesquisas posteriores (Manhães \& Glória, 1980; Manhães et al., 1981) confirmaram, para os Estados do Rio de Janeiro e do Espírito Santo e Zona da Mata de Minas Gerais, a estreita correlação desse extrator $\left(R^{2}>0,70\right)$ com o acúmulo de $P$ pela cana-de-açúcar. Em função desses resultados experimentais, o IAA-PLANALSUCAR estabeleceu, por meio de grande número de experimentos de campo, uma calibração abrangente para o extrator $\mathrm{H}_{2} \mathrm{SO}_{4} 0,25 \mathrm{~mol} \mathrm{~L}^{-1}$, chegando, então, à recomendação de adubação fosfatada para canade-açúcar, nos estados do Sul e Sudeste do País.

No Estado de São Paulo, a partir de revisão de literatura feita por Raij (1978), deu-se início ao estudo sistemático da resina de troca aniônica, para avaliar a disponibilidade de $P$ para diferentes culturas e, entre elas, a cana-deaçúcar. Esse extrator é atualmente o mais utilizado para análise do P disponível do solo naquele estado. Por outro lado, nos outros Estados, Mehlich-1 é o extrator oficialmente adotado para avaliar a disponibilidade de $\mathrm{P}$ para a cultura da cana-de-açúcar.

Assim, os diferentes extratores utilizados para avaliarem a disponibilidade de $\mathrm{P}$ são responsáveis por consideráveis variações de valores de nível crítico para $\mathrm{P}$, como $30 \mathrm{mg} \mathrm{dm}^{-3}$, encontrado por Bittencourt et al. (1978) e 9 $\mathrm{mg} \mathrm{dm}{ }^{-3}$, determinado por Marinho \& Albuquerque (1978). Outros trabalhos realizados levaram em consideração variáveis econômicas para o estabelecimento de níveis críticos de P no solo (Manhães et al., 1981; Rodella et al., 1981; Zambello Júnior et al., 1981). Essas variações são consequências das variadas características físicas, químicas e mineralógicas dos solos. Principalmente, quando se utilizam, para estimativa do P disponível, extratores que apresentam comportamento diferente em função dessas características, como o Mehlich-1, muito utilizado em muitas regiões produtoras de cana-de-açúcar, no país, como no Nordeste. Em Pernambuco, apesar da diversidade de regiões e solos, o Mehlich-1 é o extrator recomendado na avaliação da disponibilidade de P (IPA, 2008).

Poucas são as informações de pesquisa que discorrem sobre temas como disponibilidade de P e níveis críti- 
cos em cana-de-açúcar, principalmente em experimentações de campo. Elas foram mais direcionadas para outras gramíneas, como arroz (Bertoni et al., 2003), capimmombaça, capim-marandu e capim-andropogon (Mesquita et al., 2004), milho (Bedin et al., 2003) e braquiaria (Silva et al., 2004).

Assim, este trabalho teve o objetivo de determinar os níveis críticos de $\mathrm{P}$ em diferentes solos, utilizando-se diferentes extratores químicos, considerando-se a quantidade e a qualidade da fração argila; e o de relacionar características físicas, químicas e mineralógicas dos solos com os níveis críticos de $\mathrm{P}$, visando a subsidiar a recomendação de fertilizantes fosfatados em Pernambuco.

\section{MATERIAL E MÉTODOS}

Os experimentos foram conduzidos, em condições de campo, na Zona da Mata do Estado de Pernambuco, em áreas de empresas do setor sucroalcooleiro e da Estação Experimental de Cana-de-açúcar de Carpina (EECAC), da UFRPE, onde predominam solos muito intemperizados, de diferentes composições físicas, químicas e mineralógicas (Tabela 1). Nessas áreas, as características edafoclimáticas são distintas, conforme detalhadas por Koffler et al. (1986), que caracterizaram a Zona da Mata em cinco regiões edafoclimáticas: Norte, Litoral Norte, Centro, Sul e Litoral Sul.

Selecionadas as cinco áreas para instalação dos experimentos de campo, foram retiradas 30 amostras simples dos solos, na profundidade de 0,0-0,2 m, para formar uma amostra composta por área; em seguida, essas amostras compostas foram secas ao ar e passadas em peneira de 2 $\mathrm{mm}$ de malha, com o objetivo de caracterizá-las física e quimicamente. A identificação dos solos das áreas a serem avaliadas encontra-se na Tabela 1.

Os solos foram caracterizados fisicamente por sua granulometria, densidade do solo, densidade das partículas, capacidade de campo, ponto de murcha permanente e condutividade hidráulica saturada, segundo os métodos recomendados pela Embrapa (1997) (Tabela 2). Quimicamente, os solos foram caracterizados pelo $\mathrm{pH}\left(\mathrm{H}_{2} \mathrm{O}\right) ; \mathrm{pH}$ (KCl) $1 \mathrm{~mol} \mathrm{~L}^{-1}$; Ca, Mg e Al, extraídos com KCl 1 mol L-1; $\mathrm{H}+\mathrm{Al}$, determinado por acetato de cálcio $0,5 \mathrm{~mol} \mathrm{~L}^{-1} ; \mathrm{P}, \mathrm{K}$, $\mathrm{Na}, \mathrm{Cu}, \mathrm{Fe}, \mathrm{Zn}$ e Mn, por Mehlich-1; carbono orgânico, por combustão úmida com dicromato de potássio
(Embrapa, 1997). $\mathrm{O} \mathrm{Ca}^{2+}, \mathrm{Mg}^{2+} \mathrm{e} \mathrm{Al}^{3+}$ foram extraídos por $\mathrm{KCl}$ 1,0 mol L-1; o P e o K, por Mehlich-1; e o (H+Al), por acetato de cálcio 0,5 $\mathrm{mol} \mathrm{L}^{-1}$, conforme Embrapa (1997). O $\mathrm{Ca}^{2+}$ e o $\mathrm{Mg}^{2+}$ foram determinados por espectrometria de absorção atômica; o $\mathrm{K}^{+}$, por fotometria de chama, e o P, por espectrofotometria, conforme Braga \& Defelipo (1974). $\mathrm{O} \mathrm{Al}^{3+}$ e o $(\mathrm{H}+\mathrm{Al})$, por titulometria; o $\mathrm{C}$, por combustão úmida com dicromato de potássio.

O P-rem, que é a concentração de $\mathrm{P}$ da solução de equilíbrio, após agitação, durante $1 \mathrm{~h}$, da TFSA (terra fina seca ao ar) com solução de $\mathrm{CaCl}_{2} 10 \mathrm{mmol} \mathrm{L}^{-1}$, contendo $60 \mathrm{mg} \mathrm{L}^{-1}$ de $\mathrm{P}$, na relação de 1:10, foi determinado de acordo com Alvarez V. et al. (2000).

Para a avaliação da CMAP, as amostras de cada solo receberam doses de P, com base no valor de P-rem (Alvarez $\&$ Fonseca, 1990). Amostras de TFSA de cada solo foram saturadas com soluções de concentrações crescentes de $\mathrm{P}$ em $\mathrm{CaCl}_{2} 10 \mathrm{mmol} \mathrm{L}^{-1}$, adotando-se a isoterma de Langmuir para estimar a CMAP (Tabela 2).

Foi realizada análise mineralógica da fração argila dos solos, por difratometria de raios-X (Whitting \& Allardice, 1986), e a identificação dos minerais predominantes (Tabela 3). Foram determinados Fe amorfo e cristalino, extraídos por oxalato ácido de amônio (Feo) e ditionito - citrato - bicarbonato de sódio (Fed), (Mehra \& Jackson, 1960; Schwertmann \& Taylor, 1989) e determinados por espectrometria de absorção atômica (Tabela 3).

Os tratamentos consistiram em sete níveis de P, determinados em função da CMAP (Tabela 4). O delineamento experimental foi em blocos ao acaso, com quatro repetições, totalizando 28 parcelas experimentais. Cada parcela foi constituída por sete linhas com dez metros de comprimento, utilizando-se como espaçamento $1 \mathrm{~m}$ entre linhas. As doses de $\mathrm{P}$ foram aplicadas no sulco de plantio e tiveram como fonte superfosfato triplo.

Foram utilizadas as variedades RB863129, nos solos PAdx (EECAC), LAd (Cucaú), GXve (Salgado) e EKo (Santa Teresa); e a RB855536, no PAd (Pumaty). Estas variedades são cultivadas comercialmente na região e foram desenvolvidas pelo Programa de Melhoramento Genético da Cana-de-Açúcar (PMGCA), das Universidades Federais integrantes da Rede Interuniversitária para Desenvolvimento do Setor Sucroalcooleiro (Ridesa), e suas características foram descritas por Arizono et al. (1999) e Simões Neto et al. (2005).

Tabela 1 - Identificação dos solos e localização dos experimentos de campo

\begin{tabular}{lcc}
\hline Identificação/classe & Localização & Municípios \\
\hline PAdx - Argissolo Amarelo distrocoeso & EECAC & Carpina/PE \\
LAd - Latossolo Amarelo distrófico & Usina Cucau & Rio Formoso/PE \\
PAd - Argissolo Amarelo distrófico & Usina Pumaty & Joaquim Nabuco/PE \\
GXve - Gleissolo Háplico eutrófico & Usina Salgado & Ipojuca/PE \\
EKo - Espodossolo Humilúvico órtaco & Usina Santa Tereza & Goiana/PE \\
\hline
\end{tabular}

Rev. Ceres, Viçosa, v. 58, n.6, p. 802-810, nov/dez, 2011 
A correção dos solos foi realizada com calcário dolomítico e baseada na recomendação para Pernambuco (IPA, 2008), sendo de 3,0; 2,0 e 2,0 t ha-1 de calcário para os solos LAd, PAd e GXve, respectivamente. Os solos PAdx e EKo, pelos resultados apresentados das análises químicas, não necessitaram de correção.

O manejo dos solos consistiu em preparo e sulcação mecanizados, com grade aradora, grade niveladora e sulcador de aivecas de duas linhas. As adubações nitrogenada e potássica foram baseadas na recomendação para cana planta em Pernambuco (IPA, 2008). Todo N e K foram aplicados no fundo do sulco de plantio, juntamente com as doses de P. Para N, a dose padrão, em todos os experimentos foi de $40 \mathrm{~kg} \mathrm{ha}^{-1}$, utilizando-se como fonte o sulfato de amônio. Para K, utilizaram-se $100 \mathrm{~kg} \mathrm{ha}^{-1} \mathrm{de}$ $\mathrm{K}_{2} \mathrm{O}$, tendo como fonte o cloreto de potássio.

Foram coletadas três amostras simples, em cada uma das três linhas centrais da área útil das parcelas, em

Tabela 2 - Características químicas e físicas dos solos das áreas experimentais

\begin{tabular}{|c|c|c|c|c|c|}
\hline Características & PAdx & LAd & PAd & GXve & EKo \\
\hline $\mathrm{pH}\left(\mathrm{H}_{2} \mathrm{O} \quad 1: 2,5\right)$ & 5,20 & 4,10 & 4,80 & 4,90 & 5,55 \\
\hline $\mathrm{pH}(\mathrm{KCl} 1: 2,5)$ & 4,00 & 3,60 & 3,80 & 3,60 & 4,40 \\
\hline $\mathrm{H}+\mathrm{Al}\left(\mathrm{cmol}_{\mathrm{c}} \mathrm{dm}^{-3}\right)$ & 5,20 & 9,24 & 7,00 & 6,77 & 3,22 \\
\hline $\mathrm{Al}^{3+}\left(\mathrm{cmol}_{\mathrm{c}} \mathrm{dm}^{-3}\right)$ & 0,35 & 2,00 & 1,00 & 1,20 & 0,00 \\
\hline $\mathrm{Ca}^{2+}\left(\mathrm{cmol}_{\mathrm{c}} \mathrm{dm}^{-3}\right)$ & 1,90 & 0,20 & 0,90 & 5,80 & 2,20 \\
\hline $\mathrm{Mg}^{2+}\left(\mathrm{cmol}_{\mathrm{c}} \mathrm{dm}^{-3}\right)$ & 0,50 & 0,20 & 0,50 & 2,20 & 0,50 \\
\hline $\mathrm{Na}^{+}\left(\mathrm{cmol}_{\mathrm{c}} \mathrm{dm}^{-3}\right)$ & 0,01 & 0,02 & 0,03 & 0,19 & 0,04 \\
\hline $\mathrm{K}^{+}\left(\mathrm{cmol}_{\mathrm{c}} \mathrm{dm}^{-3}\right)$ & 0,07 & 0,11 & 0,10 & 0,21 & 0,07 \\
\hline $\mathrm{P}\left(\mathrm{mg} \mathrm{dm}^{-3}\right)$ & 2,83 & 0,02 & 0,70 & 4,18 & 2,17 \\
\hline CMAP $\left(\mathrm{mg} \mathrm{cm}^{-3}\right)^{1}$ & 0,33 & 0,67 & 0,78 & 0,82 & 0,10 \\
\hline P-rem $\left(\mathrm{mg} \mathrm{L}^{-1}\right)^{2}$ & 41,00 & 12,00 & 17,00 & 17,00 & 46,00 \\
\hline $\mathrm{m}(\%)$ & 12,36 & 79,03 & 39,46 & 12,50 & 0,00 \\
\hline $\mathrm{V}(\%)$ & 32,29 & 5,43 & 17,98 & 55,40 & 46,69 \\
\hline PST (\%) & 0,45 & 0,94 & 1,29 & 1,95 & 1,55 \\
\hline $\mathrm{CTC}_{\text {efetiva }}\left(\mathrm{cmol}_{\mathrm{c}} \mathrm{dm}^{-3}\right)$ & 2,83 & 2,52 & 2,53 & 9,60 & 2,81 \\
\hline $\mathrm{CTC}_{\text {total }}\left(\mathrm{cmol}_{\mathrm{c}} \mathrm{dm}^{-3}\right)$ & 7,68 & 9,76 & 8,53 & 15,17 & 6,03 \\
\hline $\mathrm{Cu}\left(\mathrm{mg} \mathrm{dm}^{-3}\right)$ & 1,05 & 0,85 & 0,35 & 2,40 & 0,60 \\
\hline $\mathrm{Fe}\left(\mathrm{mg} \mathrm{dm}{ }^{-3}\right)$ & 76,40 & 140,30 & 190,15 & 215,00 & 52,40 \\
\hline $\mathrm{Zn}\left(\mathrm{mg} \mathrm{dm}^{-3}\right)$ & 1,50 & 1,30 & 0,85 & 3,85 & 1,35 \\
\hline $\operatorname{Mn}\left(\mathrm{mg} \mathrm{dm}^{-3}\right)$ & 6,20 & 1,12 & 1,15 & 52,00 & 5,60 \\
\hline $\mathrm{C}\left(\mathrm{g} \mathrm{kg}^{-1}\right)^{3}$ & 8,90 & 9,60 & 17,90 & 13,80 & 10,70 \\
\hline Areia $\left(\mathrm{g} \mathrm{kg}^{-1}\right)$ & 785,8 & 311,6 & 539,20 & 51,60 & 855,80 \\
\hline Silte $\left(\mathrm{g} \mathrm{kg}^{-1}\right)$ & 54,6 & 9,2 & 9,20 & 209,20 & 64,60 \\
\hline Argila $\left(\mathrm{g} \mathrm{kg}^{-1}\right)$ & 159,6 & 679,2 & 451,60 & 739,20 & 79,60 \\
\hline Ds $\left(\mathrm{Mg} \mathrm{m}^{-3}\right)$ & 1,41 & 1,13 & 1,05 & 1,02 & 1,50 \\
\hline $\mathrm{Dp}\left(\mathrm{Mg} \mathrm{m}^{-3}\right)$ & 2,67 & 2,63 & 2,67 & 2,56 & 2,63 \\
\hline á $\left(\mathrm{Mg} \mathrm{m}^{-3}\right)^{4}$ & 0,47 & 0,57 & 0,60 & 0,60 & 0,43 \\
\hline $\mathrm{CC}\left(\mathrm{g} \mathrm{g}^{-1}\right)^{5}$ & 0,09 & 0,35 & 0,25 & 0,66 & 0,06 \\
\hline $\operatorname{PMP}\left(\mathrm{g} \mathrm{g}^{-1}\right)^{6}$ & 0,05 & 0,19 & 0,17 & 0,21 & 0,02 \\
\hline $\mathrm{K}_{0}\left(\mathrm{~cm} \mathrm{~h}^{-1}\right)^{7}$ & 11,60 & 6,32 & 20,92 & 1,12 & 23,05 \\
\hline
\end{tabular}

${ }^{1}$ Capacidade máxima de adsorção de P; ${ }^{2} \mathrm{P}$ remanescente; ${ }^{3}$ Carbono em forma orgânica; ${ }^{4}$ Porosidade total; ${ }^{5}$ Capacidade de campo; ${ }^{6}$ Ponto de murcha permanente; ${ }^{7}$ Condutividade hidráulica.

Tabela 3 - Identificação dos minerais predominantes nos solos, ferro amorfo e cristalino e suas relações

\begin{tabular}{|c|c|c|c|c|}
\hline \multirow{2}{*}{ Solo } & Minerais & Feo & Fed & Feo/Fed \\
\hline & \multicolumn{4}{|c|}{$\mathrm{g} \mathrm{kg}^{-1}$} \\
\hline EKo & $\mathrm{Ct}, \mathrm{Qz}, \mathrm{Gt}$ & 0,38 & 1,14 & 0,33 \\
\hline GXve & $\mathrm{Mi}, \mathrm{Ct}, \mathrm{Qz}, \mathrm{Gb}$ & 1,47 & 2,27 & 0,65 \\
\hline PAd & $\mathrm{Ct}, \mathrm{Qz}, \mathrm{Gt}, \mathrm{Gb}$ & 2,03 & 15,51 & 0,13 \\
\hline LAd & $\mathrm{Ct}, \mathrm{Qz}, \mathrm{Gt}, \mathrm{Gb}$ & 1,63 & 15,04 & 0,11 \\
\hline PAdx & $\mathrm{Ct}, \mathrm{Qz}, \mathrm{Gt}$ & 0,54 & 1,39 & 0,39 \\
\hline
\end{tabular}

$\mathrm{Ct}=$ caulinita; $\mathrm{Gb}=$ gibsita; $\mathrm{Gt}=$ goethita; $\mathrm{Qz}=$ quartzo; $\mathrm{Mi}=$ mica 
diagonal e no terço médio da área útil da parcela, formando-se uma amostra composta por parcela. Essa amostragem foi realizada 30 dias após o plantio e aplicação dos tratamentos, numa profundidade de $0,0-0,2 \mathrm{~m}$ e distante $0,25 \mathrm{~m}$ da linha da cana, para determinação do P disponível.

Nas amostras de solo coletadas, determinou-se o teor de $\mathrm{P}$ pelos extratores Mehlich-1 $\left(\mathrm{HCl} 0,05 \mathrm{~mol} \mathrm{~L}^{-1}+\mathrm{H}_{2} \mathrm{SO}_{4}\right.$ $\left.0,0125 \mathrm{~mol} \mathrm{~L}^{-1}\right)$; Mehlich-3 $\left(\mathrm{NH}_{4} \mathrm{~F} 0,015 \mathrm{~mol} \mathrm{~L}^{-1}+\mathrm{CH}_{3} \mathrm{COOH}\right.$ $0,2 \mathrm{~mol} \mathrm{~L}^{-1}+\mathrm{NH}_{4} \mathrm{NO}_{3} 0,25 \mathrm{~mol} \mathrm{~L}^{-1}+\mathrm{HNO}_{3} 0,013 \mathrm{~mol} \mathrm{~L}^{-1}+$ EDTA 0,001 mol L $\left.{ }^{-1}\right)$; Bray-1 $\left(\mathrm{HCl} 0,025 \mathrm{~mol} \mathrm{~L}^{-1}+\mathrm{NH}_{4} \mathrm{~F} 0,03\right.$ mol L ${ }^{-1}$ ) e Resina de Troca Aniônica (RTA). Os extratores químicos foram constituídos conforme métodos descritos em Mehlich (1953), Melhich (1984), Bray \& Kurtz (1945) e Amer et al. (1955), respectivamente.

Os teores de $\mathrm{P}$ do solo e a produção de cana-de-açúcar foram submetidos à análise de variância, pelo teste $\mathrm{F}$, a 5\% de probabilidade. Em seguida, realizou-se análise de regressão para os efeitos significativos. Os coeficientes dos componentes de cada modelo foram testados, escolhendo-se os modelos significativos com maior coeficiente de determinação $\left(\mathrm{R}^{2}\right)$. Com base nos modelos de regressão selecionados para produção agrícola, em função das doses de $\mathrm{P}$, foram calculadas as doses necessárias para atingir $90 \%$ da produção máxima, que foram denominadas de doses de máxima eficiência econômica (MEE). Pela substituição das doses de MEE nas equações de regressão que relacionavam os teores de $\mathrm{P}$ no solo com as doses adicionadas, foram obtidos os níveis críticos deste nutriente no solo. Foram, também, realizadas correlações entre os níveis críticos de $\mathrm{P}$ no solo determinado pelos extratores Mehlich-1, Mehlich-3, Bray-1 e RTA com características que refletem o poder tampão de fosfato dos solos, como argila, P-rem, CMAP, Feo e Fed.

\section{RESULTADOS E DISCUSSÃO}

Os dados do P extraído em função das doses aplicadas ajustaram-se a modelos lineares, nos cinco solos estudados, e as taxas de recuperação para RTA foram maiores em todos os solos (Figura 1), coerentemente com sua maior capacidade de extrair P de formas lábeis, indepen- dentemente do tamponamento do solo, conforme descreveram Silva \& Raij (1999), em pesquisa realizada em solos do Estado de São Paulo.

Os dados de produção agrícola da cana-de-açúcar em função das doses de P ajustaram-se a modelo curvilinear, do tipo raiz quadrada (Tabela 5). Com essa equação determinaram-se as doses de máxima eficiência econômica (MEE), utilizando-se a dose que correspondeu a $90 \%$ da produção máxima.

A recomendação de doses de $\mathrm{P}$ deve ser baseada na utilização de extratores que avaliem sua disponibilidade e possam estabelecer os níveis críticos do nutriente para uma determinada produção esperada. A partir desta premissa foram estabelecidos os níveis críticos para cada solo com os extratores Bray-1, Mehlich-1, Mehlich-3 e Resina de Troca Aniônica (RTA) (Tabela 6).

Os valores dos níveis críticos de P no solo variaram entre os solos e os extratores. Verificou-se que os menores valores de nível crítico ocorreram para o Mehlich-3, seguidos pelo Bray-1, Mehlich-1 e RTA, destacando-se a importância da interação solo/extrator. Estes resultados discordam dos encontrados por Bertoni et al. (2003), que estudando níveis críticos de $\mathrm{P}$ para arroz em um Organossolo Méssico, em casa de vegetação, obtiveram, para Mehlich-1, nível crítico maior do que com a RTA. Em experimentos em casa de vegetação, pela restrição do sistema radicular, as doses são maiores e a tendência é que os níveis críticos, também, tornem-se maiores, como ocorreu no ensaio de Mesquita et al. (2004), que, estudando os níveis críticos de P em três solos (LVAd, LVd e RQ) com o extrator Mehlich-1, para o estabelecimento de Capim-Mombaça, Capim-Marandu e Capim-Andropogon em vasos, verificaram que as doses de MEE de P para o Capim-Mombaça, CapimMarandu e Capim-Andropogon foram de 236, 238 e 258 $\mathrm{mg} \mathrm{dm}^{-3}$ no LVAd, respectivamente; 274,305 e $253 \mathrm{mg}$ $\mathrm{dm}^{-3}$ no LVd, respectivamente; e 94,171 e $163 \mathrm{mg} \mathrm{dm}^{-3}$ no $\mathrm{RQ}$, respectivamente. Com isso, os níveis críticos de $\mathrm{P}$ foram de 81,79 e $90 \mathrm{mg} \mathrm{dm}^{-3}$ no LVAd, respectivamente; 26, 29 e $23 \mathrm{mg} \mathrm{dm}^{-3}$ no LVd, respectivamente; e 53, 83 e 79

Tabela 4 - Níveis de fósforo em função da capacidade máxima de adsorção de fósforo e doses correspondentes aplicadas nos solos estudados

\begin{tabular}{lrrrrr}
\hline $\begin{array}{l}\text { Níveis P } \\
\text { (\% da CMAP) }\end{array}$ & \multicolumn{1}{c}{ PAdx } & LAd & PAd & GXve & EKo \\
\cline { 2 - 6 } & \multicolumn{5}{c}{ Dose de P $\left(\mathrm{kg} \mathrm{ha}^{1}\right)$} \\
\hline 0 & 0,0 & 0,0 & 0,0 & 0,0 & 0,0 \\
2 & 13,2 & 25,4 & 30,0 & 32,8 & 4,0 \\
4 & 26,4 & 51,1 & 60,2 & 65,6 & 8,0 \\
8 & 52,8 & 102,2 & 126,4 & 131,2 & 24,0 \\
12 & 79,2 & 153,3 & 189,6 & 196,8 & 32,0 \\
16 & 105,6 & 204,4 & 252,8 & 262,4 & 40,0 \\
20 & 132,0 & 255,5 & 316,0 & 328,0 & \\
\hline
\end{tabular}

Rev. Ceres, Viçosa, v. 58, n.6, p. 802-810, nov/dez, 2011 
$\mathrm{mg} \mathrm{dm} \mathrm{m}^{-3}$ no RQ, respectivamente. Os pesquisadores constataram que os menores níveis críticos de $\mathrm{P}$ ocorreram no LVd, que era o solo com mais altos teores de argila e, portanto, de maior capacidade tampão.

Neste trabalho, os extratores Bray-1, Mehlich-1 e Mehlich-3 foram responsáveis por níveis críticos menores em solos de maior capacidade tampão (Tabela 2). A média do nível crítico, quando o extrator de $\mathrm{P}$ foi Mehlich-
1, em solos mais tamponados, como LAd, PAd e GXve, foi de $6,7 \mathrm{mg} \mathrm{dm}^{-3}$, enquanto nos menos tamponados, como PAdx e EKo, foi de 14,0 mg dm-3 (Quadro 6), ou seja, para esses solos os níveis críticos foram, aproximadamente, 2 vezes maiores.

Esses dados corroboram os encontrados por Bedin et al. (2003), que, estudando fontes de P e crescimento do milho em solos com diferentes capacidades tampão de

\section{Bray-1}

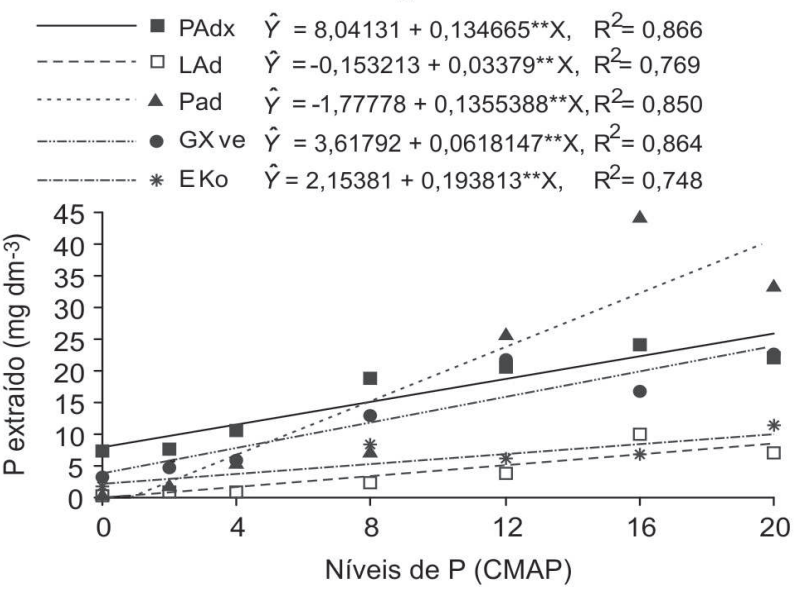

\section{Mehlich-3}
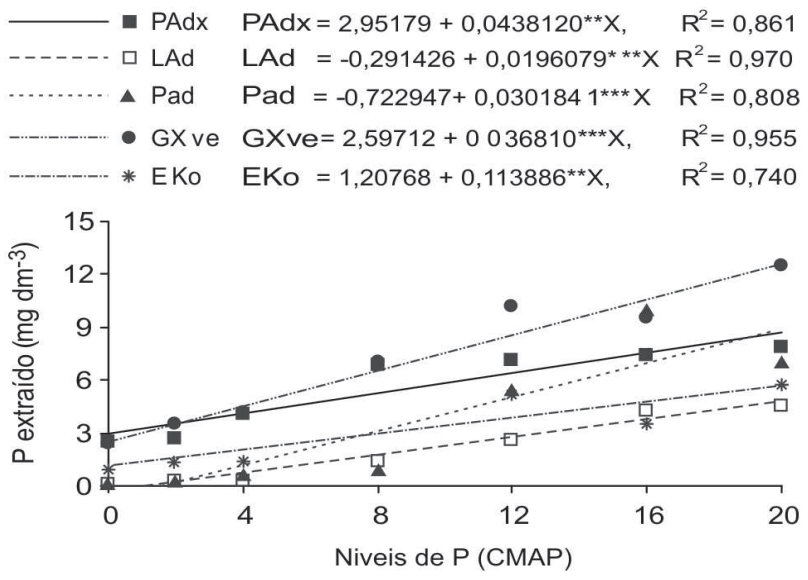

\section{Mehlich-1}

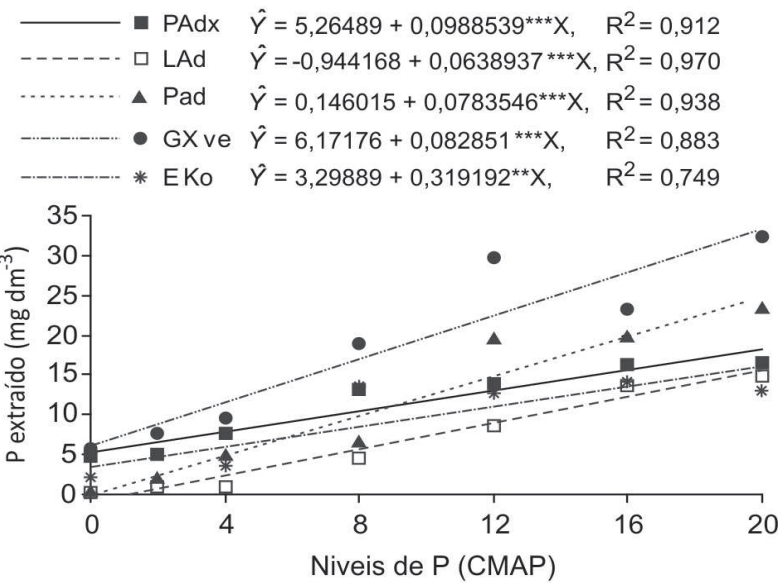

RTA

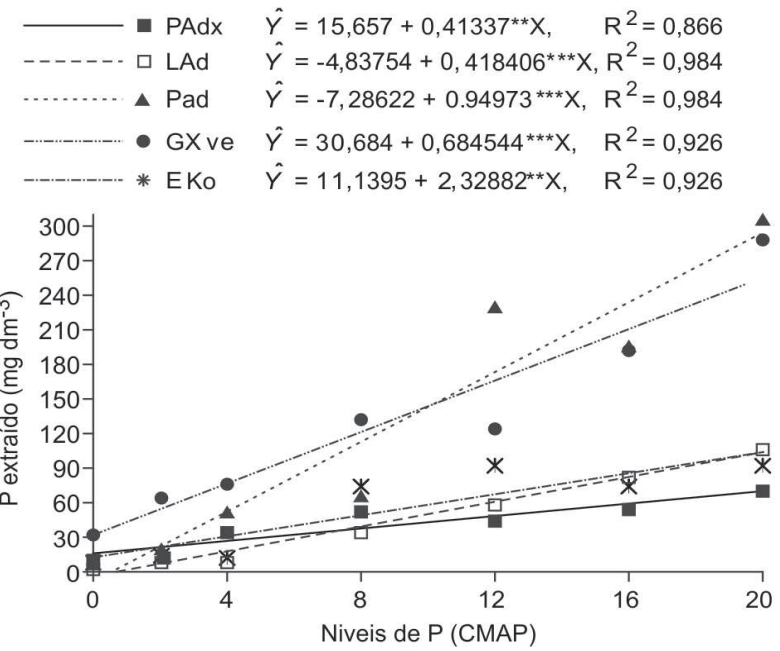

Figura 1. Equações que estimam o fósforo extraído pelos extratores Bray-1, Mehlich-1, Mehlich-3 e Resina de Troca Aniônica (RTA) em função de doses de fósforo aplicadas aos solos cultivados com cana-de-açúcar.

Tabela 5 - Equações que estimam a produção agrícola de cana-de-açúcar (TCH) como variável dependente do fósforo aplicado nos solos e a dose de máxima eficiência econômica (MEE)

\begin{tabular}{|c|c|c|c|}
\hline \multirow{2}{*}{ Solo } & \multirow{2}{*}{ Equação } & \multirow{2}{*}{$\mathbf{R}^{2}$} & \multirow{2}{*}{$\frac{\text { Dose de P (MEE) }}{\mathrm{kg} \mathrm{ha}^{-1}}$} \\
\hline & & & \\
\hline PAdx & $\hat{y}=46,2709+6,51814 * * V_{x}-0,338609 * * x$ & 0,959 & 23,4 \\
\hline LAd & $\hat{\mathrm{y}}=17,1896+9,73866 * * * \vee \mathrm{x}-0,327318 * * * \mathrm{x}$ & 0,997 & 99,9 \\
\hline PAd & $\hat{y}=70,0053+6,69611 * * * \vee x-0,21965 * * x$ & 0,988 & 61,4 \\
\hline GXve & $\hat{y}=60,3344+4,31879 * * \vee x-0,153385 * * x$ & 0,948 & 42,6 \\
\hline EKo & $\hat{y}=51,2292+15,2307 * * * \vee x-1,35216^{* * x}$ & 0,973 & 9,7 \\
\hline
\end{tabular}

** e *** significativo a 1 e $0,1 \%$, respectivamente, pelo teste $\mathrm{F}$. 
fosfato, concluíram que o fator capacidade de $\mathrm{P}$ do solo foi o principal condicionante da eficiência de utilização de $\mathrm{P}$, quando o milho foi cultivado nos solos mais tamponados.

Por outro lado, os níveis críticos determinados com a RTA não foram influenciados pelas características dos solos que refletem o fator capacidade, como teor de argila, Prem e CMAP. No GXve, que apresenta CMAP 2,5 vezes maior que o PAdx (Tabela 2), o nível crítico foi, aproximadamente, igual nos dois solos (Tabela 6). Essa característica da RTA ocorreu também com os atributos do solo que são influenciados pelo poder tampão de fosfato, ou seja, a RTA extrai P, sem apresentar variação na sua capacidade de extração com o tamponamento do solo.

Para certificar que o nível crítico de $\mathrm{P}$ no solo pode variar de acordo com extrator utilizado e com características do solo, procedeu-se à análise de correlação entre esses níveis críticos obtidos pelos diferentes extratores e as características químicas e físicas dos solos que refletem o fator capacidade (Tabela 7).

Os níveis críticos do solo, quando o extrator utilizado foi o Bray-1, não apresentaram correlação significativa para nenhuma das características do solo analisadas. Quando o extrator foi a RTA, houve a correlação significativa a $10 \%$ de probabilidade com o P-rem (Tabela 7). É, portanto, uma indicação pouco consistente da variação de níveis críticos determinados quando o extrator de $\mathrm{P}$ é a RTA, com características que refletem o fator capacidade de P. Assim, a correlação entre os níveis críticos de $\mathrm{P}$ determinados com a RTA e a CMAP contradiz a correla- ção encontrada para o P-rem (Tabela 7), fragilizando a significância do coeficiente da correlação, que parece ter ocorrido muito mais por uma variação do acaso, do que pela consistência da relação. Outros indicadores contribuem para a fragilidade dessa relação, como por exemplo, sua capacidade de extração em solos com diferentes características químicas e físicas (Figura 1).

Os níveis críticos determinados com o extrator Mehlich-3 também não apresentaram consistência para a correlação com o P-rem, porque não se confirmou com a CMAP, apesar de ter sido bem relacionado com as formas de Fe cristalinas (Feo) e amorfas (Fed) (Tabela 7). Isto significa que os componentes químicos do extrator Mehlich-3 são mais sensíveis à presença de Fe nos minerais de argila do que outros fatores responsáveis pela CMAP. Há uma predominância de Fed em relação a Feo em todos os solos (Tabela 3); porém, o comportamento da variação dos teores de Fe dos solos é bastante semelhante, o que justifica os coeficientes de correlação negativos muito próximos entre extração de P por Mehlich-3 e teores de Fe determinados com ditionito ou oxalato (Tabela 7).

Silva et al. (2004), estudando frações de $\mathrm{P}$ em Latossolos cultivados com braquiária, verificaram menores teores de $\mathrm{P}$ em solos mais tamponados e maiores teores em solos menos tamponados. Portanto, a labilidade dos teores de $\mathrm{P}$ está intimamente relacionada com características físicas e químicas que podem predizer o poder tampão de $\mathrm{P}$ dos solos. Assim, o extrator que se correlacionar com elas deverá ser mais preditivo nas estimativas dos níveis críticos.

Tabela 6 - Níveis críticos de fósforo no solo para os extratores Bray-1, Mehlich-1, Mehlich-3 e Resina de Troca Aniônica (RTA) em áreas cultivadas com cana-de-açúcar

\begin{tabular}{lcccc}
\hline & \multicolumn{4}{c}{ Nível crítico de P no solo } \\
\cline { 2 - 5 } Solo & Bray-1 & Mehlich-1 & Mehlich-3 & RTA \\
\cline { 2 - 5 } & \multicolumn{5}{c}{ mg dm $^{3}$} \\
\hline PAdx & 20,0 & 14,0 & 7,0 & 54,0 \\
LAd & 3,2 & 5,5 & 1,7 & 37,0 \\
PAd & 7,0 & 5,0 & 1,2 & 53,0 \\
GXve & 6,2 & 9,6 & 3,8 & 60,0 \\
EKo & 8,2 & 14,0 & 4,8 & 84,0 \\
\hline
\end{tabular}

Tabela 7 - Coeficientes de correlação linear entre nível crítico de fósforo no solo em cana-de-açúcar pelos extratores Bray-1, Mehlich1, Mehlich-3 e Resina de Troca Iônica (RTA) e características químicas e físicas dos solos estudados

\begin{tabular}{|c|c|c|c|c|}
\hline \multirow{2}{*}{ Característica } & \multicolumn{4}{|c|}{ Nível crítico de $\mathbf{P}$ no solo } \\
\hline & Bray-1 & Mehlich-1 & Mehlich-3 & RTA \\
\hline Argila & $-0,6571^{\mathrm{ns}}$ & $-0,940 *$ & $-0,6398^{\mathrm{ns}}$ & $-0,6300^{\mathrm{ns}}$ \\
\hline P-rem & $0,6758^{\text {ns }}$ & $0,9214^{*}$ & $0,8176 *$ & $0,7328^{\circ}$ \\
\hline CMAP & $-0,4922^{\text {ns }}$ & $-0,8217 *$ & $-0,6798^{\mathrm{ns}}$ & $-0,6560^{\mathrm{ns}}$ \\
\hline Feo & $-0,6213^{\mathrm{ns}}$ & $-0,9636 * *$ & $-0,8835^{*}$ & $-0,6409^{n s}$ \\
\hline Fed & $-0,5536^{\mathrm{ns}}$ & $-0,9328 *$ & $-0,8848 *$ & $-0,6856^{\mathrm{ns}}$ \\
\hline
\end{tabular}

$* *$, $\mathrm{e}^{\circ}$ significativo a 1,5 e $10 \%$ de probabilidade, respectivamente. ${ }^{\text {ns }}$ não significativo.

Rev. Ceres, Viçosa, v. 58, n.6, p. 802-810, nov/dez, 2011 
Os solos LAd, PAd e GXve apresentaram alta CMAP e baixo P-rem (Tabela 2), portanto, elevado poder tampão de P, condizente com suas mineralogias (Tabela 3). Verificou-se que possuem gibsita, enquanto este mineral não está presente no PAdx e no EKo, solos com menor CMAP e maior P-rem. Bahia Filho et al. (1983), estudando solos do Brasil Central, consideraram a goethita como principal componente da fração argila responsável pela adsorção de P. Neste trabalho, registrou-se a presença de goethita em todos os solos, com exceção do GXve que só apresentou gibsita em sua composição mineralógica, porém foi o solo que apresentou a maior CMAP (Tabela 2).

O Mehlich-1 apresentou correlações significativas para todas as características analisadas. Portanto, o nível crítico de P no solo, obtido quando o extrator foi o Mehlich1 , foi o que melhor se correlacionou com as características químicas e físicas dos solos. Isto reflete a variabilidade do extrator em relação ao poder tampão de fosfato dos solos e confirma a necessidade de se alterar a recomendação de adubação fosfatada, em regiões onde essas características não são consideradas, como por exemplo, na zona canavieira do Estado de Pernambuco. Embora seja o teor de argila uma análise mais universalizada do que o Prem, a indicação dessa característica como precursora da disponibilidade de $\mathrm{P}$ parece mais adequada, além da maior facilidade operacional, principalmente para as atividades rotineiras de um laboratório de fertilidade do solo.

\section{CONCLUSÕES}

A RTA extraiu mais P indistintamente, independentemente do solo.

Os níveis críticos de $\mathrm{P}$ no solo foram dependentes de características químicas e físicas que refletem o poder tampão de fosfato dos solos, quando o extrator para avaliação do P disponível foi o Mehlich-1.

Os níveis críticos de P no solo determinados pela RTA não foram dependentes de nenhuma característica química e, ou, física dos solos.

Os níveis críticos de $\mathrm{P}$ no solo determinados por Bray1 e Mehlich-3 não se correlacionaram com a CMAP.

\section{AGRADECIMENTOS}

À Diretoria e gerência agrícola das unidades agroindustriais em que foram conduzidos os experimentos: Usina Cucaú, Usina Pumaty, Usina Salgado e Usina Santa Teresa, em Pernambuco.

\section{REFERÊNCIAS}

Alvarez V. VH \& Fonseca DM (1990) Definição de doses de fósforo para determinação da capacidade máxima de adsorção de fosfatos e para ensaios em casa de vegetação. Revista Brasileira de Ciência do Solo, 14:44-55.
Alvarez V. VH, Novais RF, Dias LE \& Oliveira JA (2000) Determinação e uso do fósforo remanescente. Viçosa, Sociedade Brasileira de Ciência do Solo, p. 27-33. (Boletim Informativo, 25).

Amer T, Boulding DR, Black CA \& Duke, FR (1955) Characterization of soil phosphorus by anion exchange resin adsorption and $\mathrm{P}^{32}$ equilibration. Plant and Soil, 6:391-408.

Arizono H, Matsuoka S, Gheller ACA, Masuda Y, Hoffmann HP \& Bassinell AJ (1999) Guia das Principais Variedades RB. Araras, Universidade Federal de São Carlos, 15p. (Boletim Técnico).

Bahia Filho AFC, Braga JM, Resende M \& Ribeiro AC (1983) Relação entre adsorção de fósforo e componentes mineralógicos da fração argila de Latossolos do planalto central com diferentes características texturais e mineralógicas. Revista Brasileira de Ciência do Solo, 7:221-226.

Bedin IAE, Resende AV, Furtini Neto AE, Mendonça LA \& Vilela LCS (2003) Fontes de fósforo e crescimento do milho em solos com diferentes capacidades tampão de fosfato. Ciência \& Agrotecnologia, edição especial:1.522-1.531.

Bertoni JC, Bastos ARR, Carvalho JG \& Miranda JRP (2003) Níveis críticos de fósforo para o arroz num solo de várzea inundado em condições de casa-de-vegetação. Revista Universidade Rural, 22:45-53.

Bittencourt VC, Orlando Filho J \& Zambello Júnior E (1978) Determinations of available $\mathrm{P}$ for sugarcane in tropical soil by extraction with $\mathrm{H}_{2} \mathrm{SO}_{4} 0,5 \mathrm{~N}$. In: Congress of the International Society of Sugarcane Technologists, São Paulo. Anais, Impress. p.1.175-1.186.

Braga JM \& Defelipo BV (1974) Determinação espectrofotométrica de fósforo em extrato de solo e material vegetal. Revista Ceres, 21:73-85.

Bray RH \& Kurtz LT (1945) Determination of total, organic, and available forms of phosphorus in soils. Soil Science, 59:39-45.

CONAB (2009) Acompanhamento da safra brasileira. Brasíla, Conab. 24p.

EMBRAPA (1997) Manual de métodos de análise do solo. Rio de Janeiro, CNPS. 212p.

Koffler NF, Lima JFWF, Lacerda MF, Santana, JF \& Silva MA (1986) Caracterização edafo-climática das regiões canavieiras do Brasil. Piracicaba, IAA/PLANALSUCAR. 78 p.

IPA (2008) Recomendações de Adubação para o Estado de Pernambuco. Recife, IPA. 198p.

Manhães MS, Azeredo DF, Robaina AA \& Vieira JR (1981) Calibração do fósforo disponível para cana-de-açúcar em solos dos Estados do Rio de Janeiro, Espírito Santo e Minas Gerais (Zona da Mata). Revista Brasil Açucareiro, 27:50-56.

Manhães MS \& Glória NA (1980) Fósforo disponível para canade-açúcar (Saccharum ssp) em solos do Estado do Rio de Janeiro. Revista Brasil Açucareiro, 96:21-30.

Marinho ML \& Albuquerque GAC (1978) Calibration of extractable phosphorus in soils for sugarcane in Alagoas, Brasil. In: Congress of the International Society of Sugarcane Technologists, São Paulo. Anais, Impress. p.1.283-1.292.

Mehlich A (1953) Determination of P, Ca, Mg, K, Na and $\mathrm{NH}_{4}$. Raleigh, North Carolina Dep. of Agric. (Mimeografado).

Mehlich A (1984) Mehlich-3 soil test extractant: a modification of Mehlich-2 extractant. Communications in Soil Science and Plant Analysis, 15:1.409-1.416.

Mehra OP \& Jackson ML (1960) Iron oxide removal from clays by dithionite - citrate - bicarbonate system buffered with sodium bicarbonate. Clays and clay minerals, 7:317-327. 
Mesquita EE, Pinto JC, Furtini Neto AE, Santos PA \& Tavares VB (2004) Teores críticos de fósforos em três solos para o estabelecimento de capim-mombaça, capim-marandu e capim-andropogon em vasos. Revista Brasileira de Zootecnia, 33:290-301.

Raij B van (1978) Seleção de métodos de laboratório para avaliar a disponibilidade de fósforo em solos. Revista Brasileira de Ciência do Solo, 2:1-9.

Rodella AA, Zambelo Júnior E \& Orlando Filho J (1981) Calibração das análises de fósforo e potássio no solo em cana-de-açúcar. In: Reunião Brasileira de Ciência do Solo, Salvador. Anais, Sociedade Brasileira de Ciência do Solo. p.220-229.

Rossi C (1995) Crescimento e nutrição do braquiarão em Latossolo dos Campos das Vertentes (MG) sob influência da calagem e fonte de fósforo. Dissertação de Mestrado. Lavras, Universidade Federal de Lavras. 65p.

Schwertmann U \& Taylor RM (1989) Iron oxides. In: Bigham JM, Dixon JB, Milford MH, Roth SB \& Weed SB (Eds.). Minerals in soil environments. New York, Soil Sience Society of America. p. $379-438$

Silva EMB, Freire FJ, Santos MVF, Silva TJA \& Freire MBGS (2004) Níveis Críticos de Fósforo para Brachiaria brizantha e Suas Relações com Características Físicas e Químicas em Solos de Pernambuco. Revista Brasileira de Ciência do Solo, 28:281-288.
Silva FC (1991) Avaliação da disponibilidade de fósforo por diversos extratores em solos cultivados com cana-de-açúcar (Saccharum spp). Dissertação de Mestrado. Piracicaba, Universidade de São Paulo, Escola Superior de Agricultura Luiz de Queiroz. 165p.

Silva FC \& Raij B van (1999) Disponibilidade de fósforo em solos avaliada por diferentes extratores. Pesquisa Agropecuária Brasileira, 34:267-288

Simões Neto DE \& Melo LJOT (2005) Lançamento de novas variedades RB de cana-de-açúcar. Recife, UFRPE/Impressa Universitária. $28 \mathrm{p}$.

Strauss E (1954) Determinação do fósforo assimilável em solos de Pernambuco. In: Reunião Brasileira de Ciência do Solo, Recife. Anais, Sociedade Brasileira de Ciência do Solo. p.512-521.

Whitting LD \& Allardice WR (1986) X-ray diffraction techniques. In: Klute A (Ed.). Methods of soil analysis. Physical and mineralogical methods. Madison, Soil Science Society of America. p.331-359.

Zambello Júnior E, Orlando Filho J \& Rodella AA (1981) Recomendação de adubação fosfatada para cana-de-açúcar através da análise química do solo. Revista Brasil Açucareiro, 49:38-43. 